\title{
The multilayer structure of protons
}

\author{
I. M. Dremin ${ }^{\mathrm{a}}$ \\ Lebedev Physical Institute, Moscow 119991, Russia
}

Received: 5 January 2020 / Accepted: 10 February 2020 / Published online: 23 February 2020

(c) The Author(s) 2020

\begin{abstract}
It is argued that the dynamics of the elastic scattering of high-energy protons at intermediate transferred momenta changes with the energy increase. It evolves from the multiple scattering at the external layer for energies about $10 \mathrm{GeV}$ to the double scattering at the two subsequent layers within the colliding protons for energies about $10 \mathrm{TeV}$. The active role of the overlap function in the unitarity condition is emphasized in this context.
\end{abstract}

The collaboration TOTEM published the data on the differential cross section of elastic scattering of protons at the energy $13 \mathrm{TeV}[1,2]$. It decreases approximately exponentially (with slight oscillations as seen in the insert in Fig. 1)

$d \sigma / d t \propto \exp [-B|t|] ; \quad B \approx 20.4 \mathrm{GeV}^{-2}$

at small transferred momenta $0.04<|t|=2 p^{2}(1-\cos \theta)<$ $0.2 \mathrm{GeV}^{2}$ (where $p$ is the momentum of colliding protons and $\theta$ is their scattering angle). The exponent $B$ shows the size $R$ of the scattered protons $\left(B \approx R^{2}\right)$. It increases logarithmically with the energy increase in accordance with many theoretical models. The dip at $|t|=0.47 \mathrm{GeV}^{2}$ is usually interpreted as a consequence of zero value of the imaginary part of the amplitude at that point.

More surprising is the behavior of the cross section at somewhat larger transferred momenta $0.7<|t|<$ $3.83 \mathrm{GeV}^{2}$ (see Fig. 8; Tables 9, 10 in [1]). It shows also the exponential decrease albeit with the much smaller exponent

$d \sigma / d t \propto \exp [-C|t|] ; \quad C \approx 4.3 \mathrm{GeV}^{-2}$,

In analogy with the spatial interpretation of the exponent $B$, one is tempted to assume that this exponent gives a hint at a new deeply positioned layer inside the protons as was first claimed in Refs. [3,4] and further supported in Refs. [5-7]. In this regard, it reminds of the Rutherford discovery of the nuclei inside atoms.

a e-mail: dremin@lpi.ru (corresponding author)
From the early days of Yukawa's prediction of pions, the spatial size of protons was ascribed to the pionic cloud surrounding their centers. The pion mass sets the scale of the size in the order of $1 \mathrm{fm}=10^{-13} \mathrm{~cm}$. Numerous low-energy experiments using different methods confirmed this estimate with values of the proton radius ranging from 0.84 to 0.88 $\mathrm{fm}$. The very external shell of a proton is usually described as formed by single virtual pions as the easiest color-neutral particle constituents. That is why the one pion exchange model was first proposed [8] for the description of the peripheral interactions of hadrons. It initiated numerous multiperipheral (multireggeon) models.

The internal layers of protons must contain more massive constituents and be responsible for scattering at larger angles. Their study at larger transferred momenta asks for heavier exchanged objects and higher energies. As a particular example, let us mention the model of three-layered protons proposed in Refs. $[9,10]$. The internal layer is ascribed to $\omega$-exchanges, and the central layer consists of three quarks with a junction. The special formfactors are used for these layers with 17 adjustable parameters. However, the fits of experimental data obtained in the model are still not very successful so that it can be considered just as one of theoretical attempts. The change of the pressure inside the proton layers from the attractive one at the periphery (strongest at about $0.9 \mathrm{fm}$ ) to the repulsive one at the central regions (less than $0.6 \mathrm{fm}$ ) was found in Ref. [11]. Some intriguing features of the developing hollow at small impact parameters were noticed recently $[12,13]$ in the spatial image of proton interactions profile at $13 \mathrm{TeV}$. That could also be a signature of some new structures revealed at high energies.

The layer structure of protons can be at the origin of different behavior of their scattering outside the diffraction cone with the rise in collision energies. At the energies about 10 $\mathrm{GeV}$ the differential cross section behaved there differently than shown by Eq. (2) at $13 \mathrm{TeV}$, namely,

$d \sigma / d t \propto \exp [-b \sqrt{|t|}] \approx \exp [-b p \theta]$. 
Fig. 1 The differential cross section of elastic scattering of protons at $13 \mathrm{TeV}$ shown in [2] and discussed in $[3,4]$. The upright insert demonstrates slight oscillations within the diffraction cone. Another insert contains some theoretical predictions outside the diffraction cone
- Large amount of data (trigger rate $50 \times$ w.r.t. Run I)

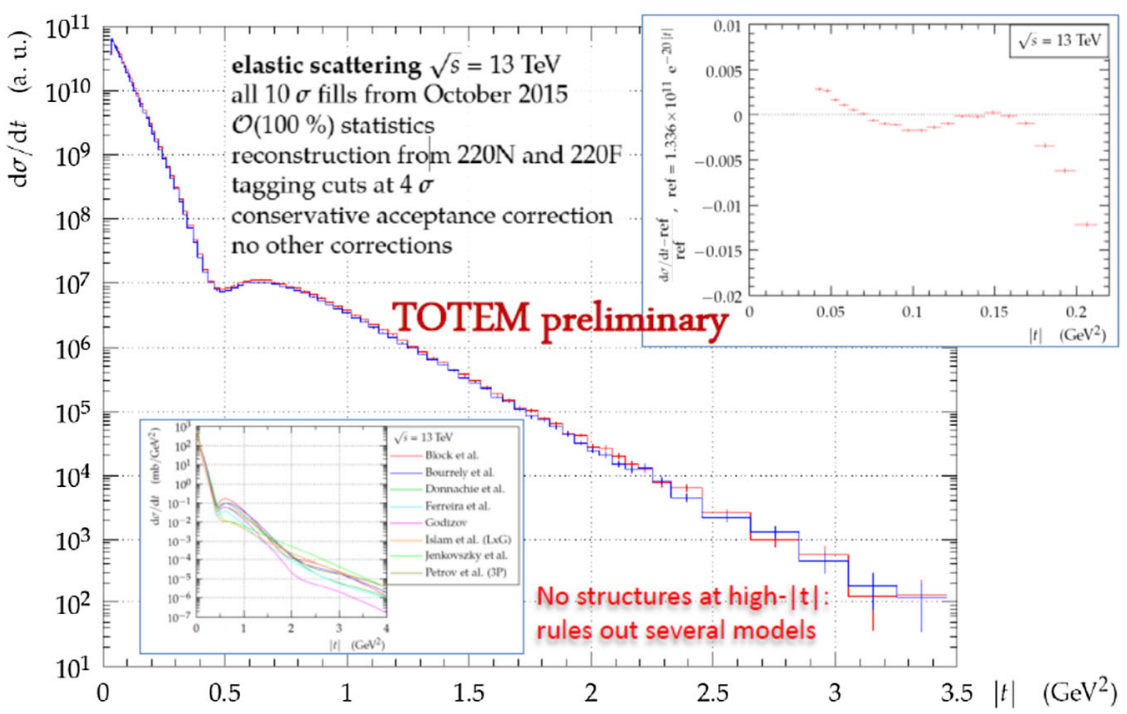

That was called the Orear regime by the name of its discoverer [14]. The $\sqrt{|t|}$-exponential behavior outside the diffraction cone replaced the $t$-exponential one inside it.

However, the experimental data at $13 \mathrm{TeV}$ show that the above $\theta$-exponential regime at intermediate transferred momenta outside the diffraction cone changes drastically again to the much faster $t$-exponential ( $\theta$-Gaussian) decline of Eq. (2). It is similar to the behavior in the diffraction cone but with the much smaller exponent. If interpreted in terms of the spatial sizes, it looks as if another smaller size starts playing a role. This puzzle seems especially intriguing because the $\sqrt{|t|}$-dependence of Eq. (3) was derived as a consequence of the unitarity condition [15-17] and of the multiple Pomeron exchange [18]. Then the general question arises of what happens with the unitarity and what mechanism is at work at $13 \mathrm{TeV}$.

The unitarity condition $S S^{+}=1$ imposed on the $S$-matrix can be written in terms of the scattering amplitudes as [17]

$$
\begin{aligned}
\operatorname{Im} A(p, \theta)= & \frac{1}{32 \pi^{2}} \int d \cos \theta_{1} d \cos \theta_{2} \\
& \times \frac{\operatorname{Re} A\left(p, \theta_{1}\right) \operatorname{Re} A\left(p, \theta_{2}\right)+\operatorname{Im} A\left(p, \theta_{1}\right) \operatorname{Im} A\left(p, \theta_{2}\right)}{\sqrt{\left[\cos \theta-\cos \left(\theta_{1}+\theta_{2}\right)\right]\left[\cos \left(\theta_{1}-\theta_{2}\right)-\cos \theta\right]}} \\
& +F(p, \theta) \\
= & \int d \Phi_{2} A A^{*}+\Sigma_{n} \int d \Phi_{n} M_{n}(p, 0) M_{n}^{*}(p, \theta) .
\end{aligned}
$$

The first terms in both sums denote the contributions from purely elastic rescattering to the imaginary part of the elastic amplitude $\operatorname{Im} A$. The second terms (called the overlap function $F$ [19]) correspond to the interference of the inelastic amplitudes $M_{n}$ of $n$-particle production with initial and final two-particle states turned at the angle $\theta$. The integration region is given by the conditions $\left|\theta_{1}-\theta_{2}\right| \leq \theta ; \quad \theta \leq \theta_{1}+\theta_{2} \leq 2 \pi-\theta$.

The unitarity condition (4) is usually considered with two assumptions that the contributions of the real parts to the integral term and the overlap function are small compared to the role of imaginary parts in the integral. First assumption stems from smallness of $\operatorname{Re} A$ in the diffraction cone. It is small compared to $\operatorname{Im} A$ in the forward direction [20] and possesses zero within the cone [21]. Intuitively, the overlap function is also small because inelastic processes at high energies proceed within narrow cones along the colliding particles. Therefore the overlap of these cones must be small since the two-particle states of the overlap function in the unitarity condition are considered at the large transferred momentum. This is indicated by the arguments 0 and $\theta$ of the inelastic matrix elements $M_{n}$ in (5) which show that the initial headon collision leading to the final two-particle state is turned at the angle $\theta$.

If these assumptions are accepted and the corresponding terms are omitted, the unitarity condition becomes the nonlinear integral equation for the imaginary parts of the amplitude $\operatorname{Im} A$. Its iterative solutions were attempted $[15,16]$. They lead to the Orear regime. However, the obtained values of the exponent did not agree with experimental ones. Too many iterations were required so that the iteration series started to contradict the unitarity. The model with many Pomeron exchanges was also exploited [18]. It also leads to the qualitatively correct Orear behavior albeit with somewhat different main exponent and additional (unobserved!) oscillations. Nowadays it fails to explain the t-exponential behavior in the large-t region.

However, the non-linear equation can be actually transformed to the linear one [17]. It is possible because the main contribution to the integral term comes from asymmetrical 
angles. Thus, one of $\operatorname{Im} A$ can be inserted from the diffraction cone replacing it by $\sqrt{d \sigma / d t}$ from Eq. (1) while another one is at the angle close to $\theta$. The final solution is of the Orear type. The exponents happened to be close to their experimental values at energies about $10 \mathrm{GeV}[17,22]$. Even though the direct iterative approach is not necessary here, one is tempted to consider the Orear regime as a consequence of some (may be, finite number) iterations, i.e., as rescatterings induced by the external layers of protons.

Surely, the unitarity should be valid at any energy. The $t$-exponential decrease of the differential cross section at 13 $\mathrm{TeV}$ can be explained by the new mechanism of scattering at intermediate transferred momenta. Protons are able to penetrate inside each other deeper at higher energies and higher transferred momenta. Thus in place of the multiple rescattering of the external layers in the GeV-energy range the successive double scattering of the external and deeper layers happens at the $\mathrm{TeV}$ energies. Both scatterings are Gausian ones in terms of the angles with exponents corresponding to two different internal sizes. In the unitarity relation (4) one of $\operatorname{Im}\left(p, \theta_{i}\right)$ should be used as a $\theta_{i}$-Gaussian exponent with the size of the external layer $B$ and another one as a $\theta_{i}$-Gaussian exponent with the size of the internal layer $\beta$. In experiment, one would observe the $t$-exponential decrease of the differential cross section with the exponent

$C=\frac{B \beta}{B+\beta}$.

Using the experimental values of $B=20.4 \mathrm{GeV}^{-2}$ and $C=4.3 \mathrm{GeV}^{-2}$ at $13 \mathrm{TeV}$ (see Fig. 1) one can find the exponent of the internal layer $\beta \approx 5.4 \mathrm{GeV}^{-2}$. In terms of the spatial size the internal layer is concentrated at the radius near $0.45 \mathrm{fm}$, twice smaller than the external size. Thus we claim that the scattering at $13 \mathrm{TeV}$ reveals the second layer inside the protons.

It is claimed in Ref. [1] that at the largest transferred momenta from 2.1 to $4 \mathrm{GeV}^{2}$ the power law favored by the quark counting rules can be adopted with the exponent of the order of 10 . However, the measured angles are less than $3 \cdot 10^{-4}$, i.e., too small for these rules to be applicable there. Moreover, the exponential fit (2) works well in a wider range from 0.7 to $3.8 \mathrm{GeV}^{2}$.

What concerns the unitarity condition, the validity of the assumptions about the overlap function and the contribution of the real parts of the elastic amplitude can not be proved if only experimental data are available. They provide $d \sigma / d t \propto(\operatorname{Re} A)^{2}+(\operatorname{Im} A)^{2}$ but not the real and imaginary parts separately. Some theoretical help is necessary to get the forward values of the real and imaginary parts of the amplitude and the guesses about the zero value of the real part inside the diffraction cone. Additional models and approximations are needed for more detailed description of the amplitudes. The good fits of experimental data and knowl- edge of the energy behavior of real and imaginary parts of the elastic scattering amplitude separately are claimed in $[13,23]$. That can be used to verify the validity of the assumptions used for the above treatment of the unitarity relation. One would guess that the overlap function starts playing the more important role at higher energies. Theoretically it could be ascribed to the phases of the matrix elements of inelastic processes $M_{n}$ in (4). Their interference could suppress the $\sqrt{|t|}$-exponent down to the observed t-exponential regime. The work is in progress.

Concluding, we argue that at higher energies the deeper layers of protons enter the game for protons scattered outside the diffraction cone. The multiple scattering inside the external layer observed there at $\mathrm{GeV}$-energies is replaced by a common effect of the double scattering due to the external and internal layers at $\mathrm{TeV}$-energies. That restores the $t$ exponential decrease of the differential cross section outside the diffraction cone at $\mathrm{TeV}$-energies which replaces the $\sqrt{|t|}$ exponential Orear-behavior at GeV-energies.

Acknowledgements I am grateful for support by the RAS-CERN program.

Data Availability Statement This manuscript has no associated data or the data will not be deposited. [Authors' comment: This is a theoretical study and no reference to experimental data.]

Open Access This article is licensed under a Creative Commons Attribution 4.0 International License, which permits use, sharing, adaptation, distribution and reproduction in any medium or format, as long as you give appropriate credit to the original author(s) and the source, provide a link to the Creative Commons licence, and indicate if changes were made. The images or other third party material in this article are included in the article's Creative Commons licence, unless indicated otherwise in a credit line to the material. If material is not included in the article's Creative Commons licence and your intended use is not permitted by statutory regulation or exceeds the permitted use, you will need to obtain permission directly from the copyright holder. To view a copy of this licence, visit http://creativecomm ons.org/licenses/by/4.0/.

Funded by SCOAP ${ }^{3}$.

\section{References}

1. G. Antchev, P. Aspell, I. Atanasov et al. (TOTEM collaboration) arXiv: 1812.08283

2. T. Csörgö, Talk at Low-x 2017, 12-18 June 2017. Bislegli. https:// indico.cern.ch/event/609299/

3. I.M. Dremin, Phys. Usp. 61, 381 (2018)

4. I.M. Dremin, Particles 2, 57 (2019)

5. T. Csörgö, R. Pasechnik, A. Ster, Eur. Phus. J. C 79, 62 (2019)

6. T. Csörgö, R. Pasechnik, A. Ster, arXiv:1910.08817

7. S.M. Troshin, N.E. Tyurin, arXiv:2001.06260

8. I.M. Dremin, D.S. Chernavsky, JETP 38, 229 (1960)

9. M.M. Islam, Nucl. Phys. B 104, 511 (1976)

10. M.M. Islam, J. Kaspar, R.J. Luddy, Mod. Phys. Lett. A 24, 485 (2009)

11. V.D. Burkert, L. Elouadrhiri, F.X. Girod, Nature 557, 396 (2018)

12. I.M. Dremin, Phys. Usp. 58, 61 (2015) 
13. T. Csörgö, R. Pasechnik, A. Ster, Acta Phys. Pol. B Proc. Suppl. 12, 779 (2019)

14. J. Orear, Phys. Rev. Lett. 12, 112 (1964)

15. D. Amati, M. Cini, A. Stanghellini, Nuovo Cim. 30, 193 (1963)

16. V.N. Cottingham, R.F. Peierls, Phys. Rev. 137, B147 (1965)

17. I.V. Andreev, I.M. Dremin, JETP Lett. 6, 262 (1967)

18. A.A. Anselm, I.T. Dyatlov, Phys. Lett. B 24, 479 (1967)
19. L. Van Hove, Nuovo Cim. 28, 798 (1963)

20. I.M. Dremin, M.T. Nazirov, JETP Lett. 37, 198 (1983)

21. A. Martin, Lett. Nuovo Cim. 7, 811 (1973)

22. I.V. Andreev, I.M. Dremin, I.M. Gramenitsky, Nucl. Phys. B 10, 137 (1969)

23. A.K. Kohara, E. Ferreira, T. Kodama, Eur. Phys. J. C 74, 3175 (2014) 